\title{
Pop-Up Concrete: Rethinking Concrete Shell Structures
}

\author{
ROGER HUBELI \\ Syracuse University
}

This design research investigates a computational system for concrete shell structures that translates digital modeling into adjustable formwork configurations with the use of a unique fabrication technique coined 'pop-up concrete.' This design method is developed in collaboration with CEMEX, a cement manufacturer with their material scientists and engineers working with architects to expand our approach to fabrication in concrete. Typically, complex concrete structures, such as shells, require elaborate and expensive formwork and reinforcement. But with the use of advanced concrete mixes, in combination with parametric design tools that allow for the flexible manipulation of digitally fabricated formwork, it is possible to revisit complex concrete shell structures for ubiquitous, mass-customized and formally varied applications.

'Pop-up' is a method of using very thin, high performance concrete with a formwork that begins as a flat surface and is folded and popped up into a final form during the curing process. The method begins with digital modeling that is recalibrated into a 'foldable' formwork that can be infinitely altered to various configurations, depending on its material properties. 'Pop-up' has the potential to test the capacity for concrete structures to be built as parametrically varied complex geometries. The challenge is in the translation of the precise variability of digital modeling into the imprecise variations of concrete casts. This paper aims to show how 'pop-up' techniques revisit the methods of concrete shell design and construction through new computational methods and digital fabrication. The research serves as a first feasibility study with prototypes based on computational modeling that influences new fabrication techniques and identifies further challenges.

\section{INTRODUCTION}

Roof structures of thin-shell concrete, such as Felix Candela's Los Manantiales Restaurant (Arup 1963) or Heinz Isler's Tennis Centers (Chilton 2000), are a prototypical product of the twentieth century, but they were not seen much at the start of the present century (Billington 2008). Although, the introduction of digital technology in structural computation in the late twentieth century radically widened the range of possible geometries in thin shell design (Windeck 2016), the techniques of shell construction have not advanced at the same pace. For example, Mutsuro Sasaki's engineering for Toyo Ito's complex shell roof for the Cemetery in Kakamigahara uses sensitivity

\author{
JULIE LARSEN \\ Syracuse University
}

analysis based on advanced structural computation to optimize the form and material use (Sasaki 2007). However, the construction of this complex shell still required traditional formwork and reinforcement, not unlike the construction methods deployed by Candela or Isler 50 years prior. This results in the fabrication of rather complex falsework and formwork made of custom timber construction, as well as the necessary complicated reinforcement patterns.

Recent projects, such as Block Research Group or Culver \& Sarafian's concrete shells aim to create complex computational geometry, while simultaneously challenging the fabrication methods of shell construction to significantly reduce material and labor costs. Culver \& Sarafian demonstrate the use of industrial robots that are capable of generating various cast components for grid shells based on digital input to facilitate otherwise cost-prohibitive design (Culver 2017). Block Research Group uses a reusable net formwork to eliminate the typical wood falsework and formwork; thus, potentially reducing the cost of construction and making it possible to envision a wide range of anticlastic shapes (Veenendaal 2014).

Building upon the above projects, the described work seeks to translate computational advantages through the use of a 'pop-up' formwork that is folded into position; a technique that dramatically reduces material and labor while still achieving complex geometries derived from computational input. The project uses high performance, fiber reinforced, lightweight concrete that is cast onto flat formwork and folded into shape during the curing process. The technique significantly reduces the complexity of the formwork and potentially eliminates the need for mesh reinforcement to keep the profile thinner.

\section{'FOLDED' CONCRETE FORMS}

"Folding" in concrete has been a fundamental principle to increase the strength and stability of structural elements. 'Folding' increases a material's structural capacity without having to increase the amount of material used or strengthening it. Although there are many folded structures made of concrete, there has always been a schism between the material and the technique. For example, contrary to sheet metal, concrete is not inherently a flat material but a liquid that is poured to take a "folded" shape for structural benefits. Therefore, the construction of a folded concrete form requires a complex formwork 


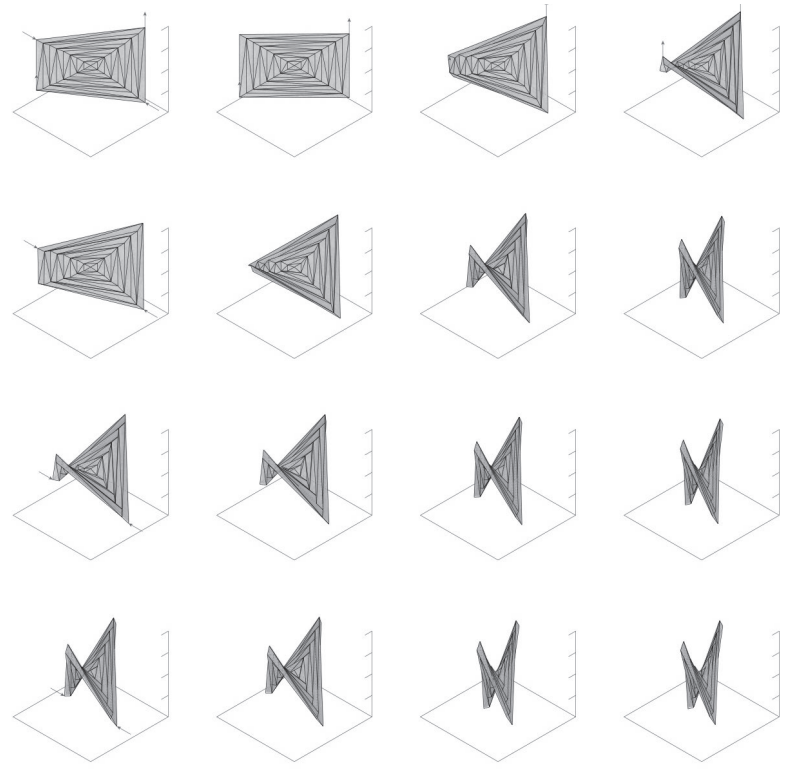

Figure 1. 'Creased shell' structure parametrically controlled in Grasshopper to determine the varying relationships between spans (distance between supports) and heights (drawing by APTUM).

that is not actually folded, but does create a folded form for the concrete to be cast in. However, with the advancement in the concrete mix designs and the improvements to the performance of fiber reinforcement, it is possible for a formwork to fold into infinite forms and positions while the concrete is still curing. This is the basis for the 'pop-up' method that casts concrete in a flat formwork and then positions the formwork into its final form in the early stages of curing. Similar to Wierzbicki-Neagu's 'Folded Architecture,' comprised of kinetic structures that can be developed into a variety of topologies (WierzbickiNeagu 2005), the 'pop-up' formwork technique can make concrete as 'deformable' and 'infinitely arrayed' as their mobile structures to provide many different types of shell topologies with a singular formwork.

The benefits to the 'pop-up' construction method, as a way to truly fold concrete, are three-fold. First, this method uses considerably less falsework and formwork to create complex geometries. Second, a singular formwork can be computationally controlled and altered after each pour to create a variety of elements that vary in height, width, or depth. Third, traditional concrete cannot be 'popped-up' because it is either initially too fluid or, once cured, becomes too brittle. Therefore, this requires reinforcement to be formed into the shape of the final form prior to casting. Since fiber reinforced concrete does not have the tedious task of shaping and positioning reinforcement within the formwork, the 'pop-up' technique can significantly reduce labor and material costs while still achieving a complex computational geometry.

\section{METHODS}

To test the described 'pop-up' techniques, a 'creased' hyperbolic paraboloid shell was used as a basis for the first prototype. The form of the prototype addresses the tension between the computational form and the needed alterations to make the form 'precise' as a formwork and the final concrete cast. Where a parametric model can achieve almost any form, many steps must but considered to achieve the same precise form in analog. The following steps articulate the process by which we achieved the translation from a parametric model to a digitally fabricated formwork for a series of initial prototypes.

\section{THE MIX}

The techniques and methods discussed pertain to the ability for new concrete technology to perform in ways not achievable 10 years ago. The concrete mix, with extremely fine aggregates, results in high performance concrete with high strength and lightweight density $(1.4 \mathrm{~kg} / \mathrm{l}$ in comparison to $2.4 \mathrm{~kg} / \mathrm{l}$ for 'standard' concrete) to create much lighter and thinner forms. The high strength of the material (after 28 days $65 \mathrm{MPa} / 9500 \mathrm{psi}$ ) offers the ability for concrete to be thin and light enough to fold into position. In addition, the lightness of the concrete also reduces the dead load of the structure and therefore helps to reduce the outward thrust at the supports. The use of plasticizer and accelerator in the mix increases fluidity and speed of curing, respectively, which allows the formwork to be moved into vertical positions within hours after the concrete is poured. And due to the high flexural strength and fast curing of the mix, a flat piece of formwork can easily be 'popped up,' folded or creased like a piece of paper into its final form after only 3 hours of curing and demolded after only 3 days.

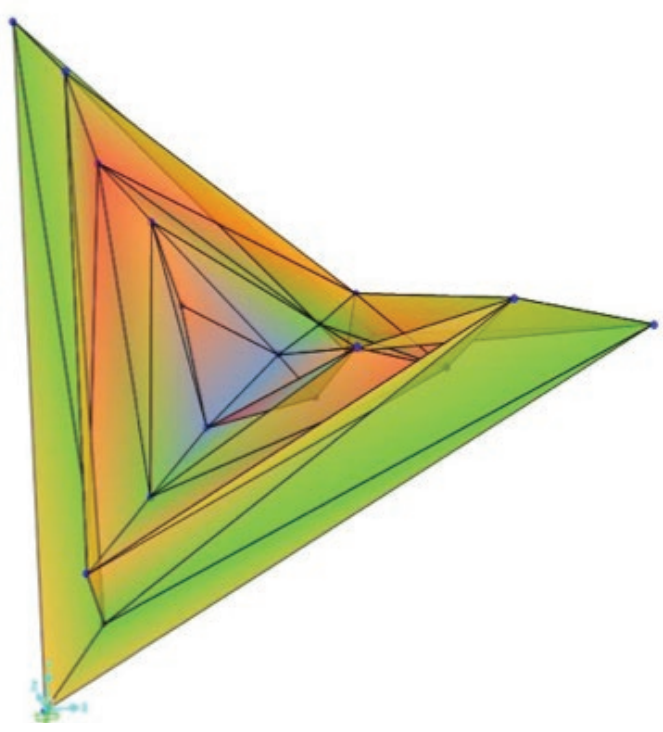

\begin{tabular}{l}
6.00 \\
5.25 \\
4.50 \\
3.75 \\
3.00 \\
2.25 \\
1.50 \\
0.75 \\
0.00 \\
-0.75 \\
-1.50 \\
-2.25 \\
-3.00 \\
-3.75 \\
\hline
\end{tabular}

Figure 2. SAP2000 structural diagram of 'creased shell' (drawing by XX). 


\section{PARAMETRIC GEOMETRY}

The shell forms were generated in grasshopper with specific coordinates that control the various degrees of change in the forms. Depending on the type of fold or 'crease' in the form, specific parameters were exaggerated or diffused in the model, such as height, width, depth, scale, distance between edges, middle and corner points, or surface alignments. These parameters were then used in varying degrees of complexity or aggregation to produce different scales and proportions of the shell structures (figure 1). In future prototypes, the points on the parametric model will be translated to the formwork with more precise measurement and accuracy using robotic coordinates to position points in space on the formwork.

\section{DYNAMIC STRUCTURAL AGGREGATION}

A singular formwork can produce a series of shell elements; resulting in an aggregated system of shells to form a larger structural framework. The intention with the formwork is to not only produce iterations of forms but to generate a more dynamic structure where a series of elements can vary in height, width, or depth with a singular formwork. The digital model also provides precise measurement of seams, edges, and boundaries so that the formwork can accurately be measured and concrete can precisely be poured into the formwork to generate the varied degrees of structural forms. It is this exercise of formal generative logic that truly utilizes one simple formwork for a multitude of varied forms. This allows for a very rapid construction of complex geometries with minimal labor and drastically reduced weight and material.

\section{STRUCTURAL ANALYSIS}

The concrete shell was preliminarily tested by the engineer in SAP2000 to check for overall stresses on the surface (figure 2). The maximum principal stress (SMAX) on the visual surface under the self-weight is small enough for the current mix design, even with some stress concentrations in the middle area due to the overall compressed form and the individual creases in the middle of the surface. The even stress distribution implies that it is possible to evenly scale up the shell without disproportionate local increases in stresses.

\section{THE FABRICATION PROCESS}

Once the geometry in grasshopper is established, the flattened form is CNC milled as a series of flat elements for the formwork. To re-establish the formwork as a dynamic surface, similar to the grasshopper model, edges and points of the formwork are defined as either 'peaks' or 'valleys' to either be pulled up or pushed down in the fabrication process. In initial prototypes, the individual elements of the formwork are hinged together on either the front or back to create the peak or valley of the creased surface. Once the formwork is hinged and flat, it is ready for the concrete to be poured into position. After the concrete is poured into the flat formwork, it can be 'popped' into the final position after just 3 hours of
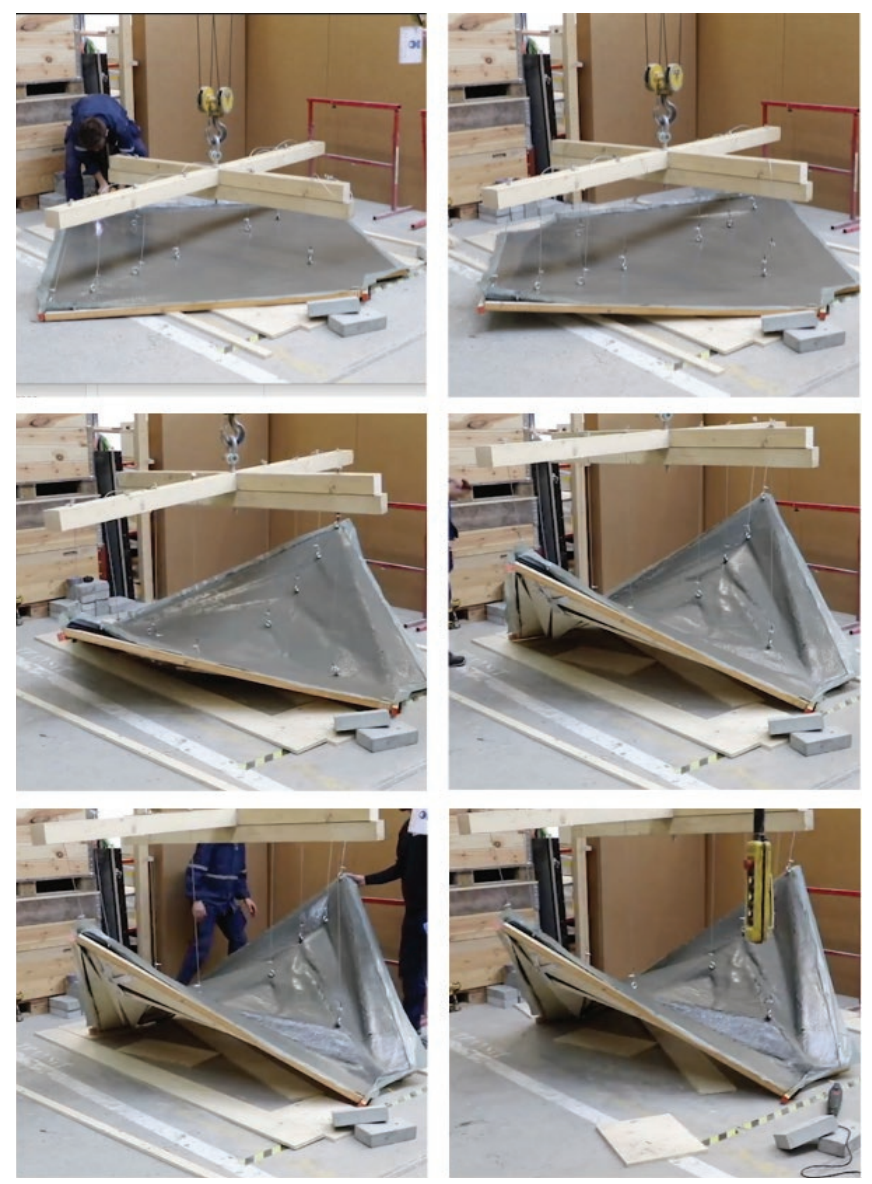

Figure 3. Step by step process of formwork from flat position to 'creased' pop-up form. Hinged wood formwork acts like fabric; able to be pushed and pulled. The concrete mix, after just 3 hours, can be pushed and pulled like fabric into a final configuration (photos by APTUM).

curing. Given the position of the hinges on either side of the formwork, similar to the computational model, the folds selfposition when the outer points of the formwork are pulled up to define the overall geometry (figure 3 ).

\section{RESULTS AND DISCUSSION}

The Pop-up techniques and prototypes resulted in four fundamental observations. First, the complex and messy relationship between digital form, mix and fabrication helps to recalibrate new fabrication strategies for computational form but also challenges the economies of labor, material flow, and production. The questions explored in this research project reflects how new fabrication techniques make it possible to reconnect with the tradition of shells as ubiquitous methods for roof construction that engineers/architects like Candela and Isler were able to popularize.

Second, Pop-Up Concrete is in the early stages but has already proven that the mix is viable for the pop-up formwork technique. The technique will become more feasible once the shell formwork increases in size. In the first attempts, the formwork was too small and became finicky 


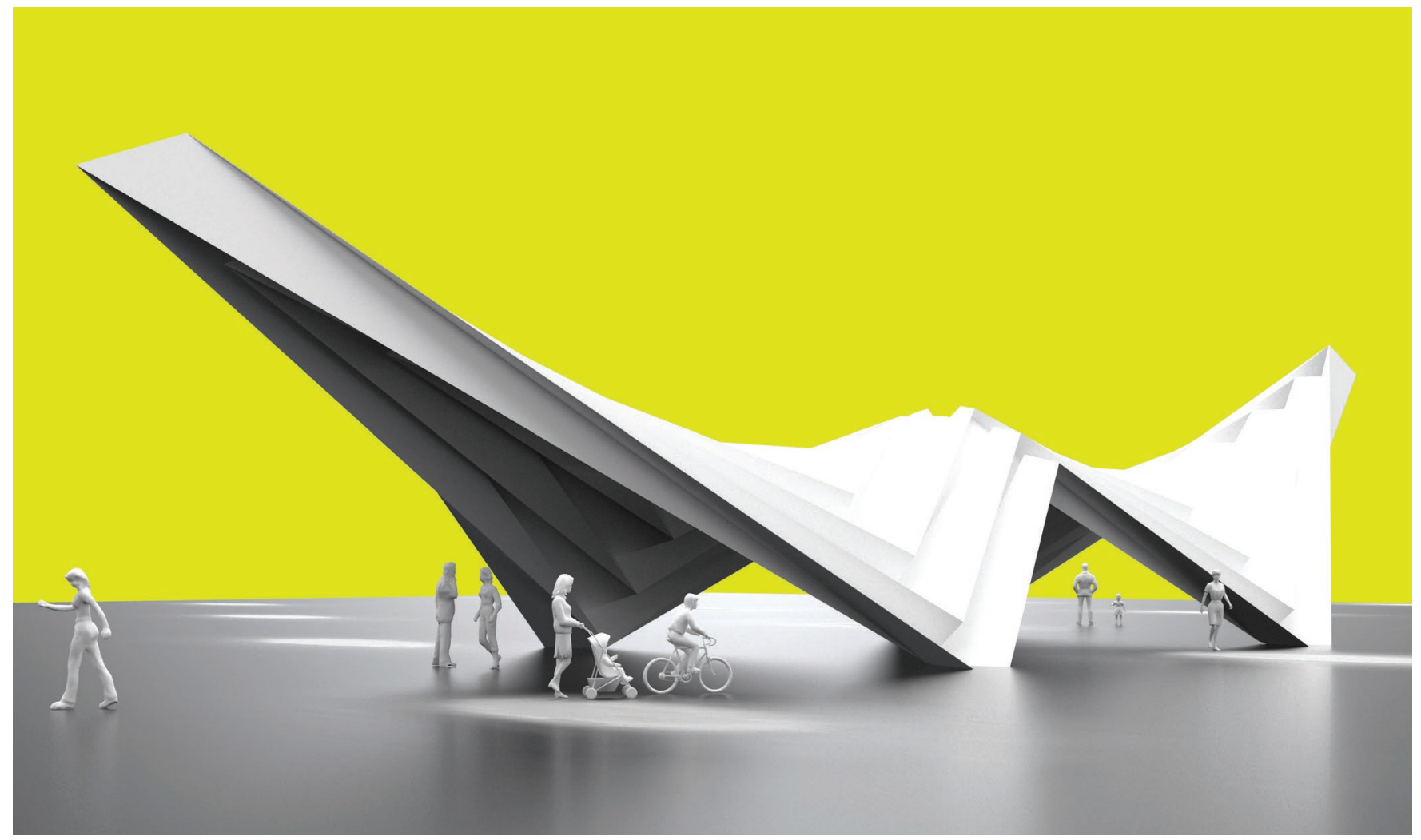

Figure 4. Exterior rendering of aggregated 'creased shells' as a larger scale structure.

to move into position. But as the size of the formwork elements increased, it was simpler to adjust the formwork with large hinges and wider gaps between elements; making the creases in the form capable of self-positioning with little friction (figure 5).

Third, the first prototypes resulted in a concrete that could easily be molded into a complex geometry without slumping and result in a form that closely simulated the computational geometry. But progress needs to be made in terms of defining precise edges, corners and connections as more forms are made to aggregate and connect to one another. More details will be required to pre-cast voids in the concrete for pin connections to attach multiple shells together. In addition, a detail is in development to ensure a proper foundation that can resist the occurring outward thrust through the use of a tension member that spans between the supports. Ideally, this member could double up as a rail to move the formwork during the pop-up process.

Lastly, the computational goals of creating a singular formwork that can vary in height, width, and depth, needs to be carefully calibrated to ensure that edges of different shell structures can precisely align without unwanted gaps between forms. The goal is to build additional shell prototypes at a larger-scale in anticipation that some details can be more accurately controlled once the form is larger and the thicknesses of the forms are more reasonable for the connections needed. This makes it possible to envision the construction of a wider range of computational geometries.

The goal of the next phase of 'Pop-Up' is to achieve a series of 'creased' shell geometries that are made using robotic arms (figure 6). The Pop-up method for the formwork with a robotic arm augmented by a gantry is an opportunity to cast more complex geometries. The introduction of more advanced digital fabrication techniques with robotics and automation will allow us to further develop the pop-up techniques at a larger scale but also to build with more precision and consistency. The precise forms derived from fabricating with the robotic arm will offer the potential for easier assembly of the concrete objects once they are aggregated.

The intention is to script the pop-up strategy and make 1:1 mock-ups with robotic arms. The intention is to make concrete forms at the 1:1 scale and assemble the pieces into a larger structure to work out assembly, connections, load, and stresses. The payload of the robotic arms are minimal compared to larger scale structures but this research will determine the viability of the technique at a scale more related to larger assemblies. Once we can determine the overall strategy, the next step will be to do the pop-up technique at a scale that could potentially be controlled by GPS guided cranes. 


\section{CONCLUSION}

Pop-Up techniques using lightweight, high-strength concrete has the potential to compete for building programs that require large spans but are relatively inexpensive and quick to construct. If the construction process for concrete can become faster to deploy larger structural frameworks, there is potential to enter into markets where concrete is commonly overlooked. One way to achieve this is through automation and robotics. The next phase to the pop-up techniques is to cast formwork that is positioned into place with robotic arms that can be more precise in locating points in space. There are untapped architectural applications that would greatly benefit by rethinking how to digitally develop a construction method from the bottom-up strategy into an aggregated, dynamic system. Shell structures that were more ubiquitous in the 50 s and $60 \mathrm{~s}$, as factories, airplane hangars, train stations, bus terminals, etc. could see a revival with the concrete pop-up method (figure 4). By rethinking the way concrete can be digitally fabricated, through flexible, pop-up structures, it has the potential to become more proliferate as years past and bring complex shell and folded structures back into the competitive field of mass-produced and mass-customized buildings.

\section{REFERENCES}

Arup, Ove and Colin Faber. Candela's Work: Candela, The Shell Builder New York: Reinhold Pub. Corp., 1963, 194-198.

Billington, David and Maria Garlock. "Thin-Shell Concrete Structures: The Master Builders," in Seven Structural Engineers the Felix Candela Lectures, ed., G. Nordenson. New York: The Museum of Modern Art, 2008, 161.

Chilton, John. Heinz Isler. London: Telford, 2000, 106.

Culver, Ronald and Joseph Sarafian. "Robotic Formwork in the MARS Pavilion: Towards The Creation Of Programmable Matter." ACADIA 2017 | Disciplines + Disruption (2017): 522

Sasaki, Mutsurō and Arata Isozaki, and Toyo Itō. Morphogenesis of Flux Structure. London: AA Publications, 2007, 87.

Veenendaal, Diederik, and Philippe Block. “Design Process for Prototype Concrete Shells Using a Hybrid Cable-Net and Fabric Formwork." Engineering Structures 75 (2014): 39-50.

Wierzbicki-Neagu, Madalina. "Unfolding Architecture - Study, Development and Application of New Kinetic Structure Topologies," ACADIA 2005: Smart Architecture (2005): 246

Windeck, Georg. "Evolutionary Structural Optimization," in Construction Matters. Brookyln, NY: Powerhouse Books, 2016, 80.

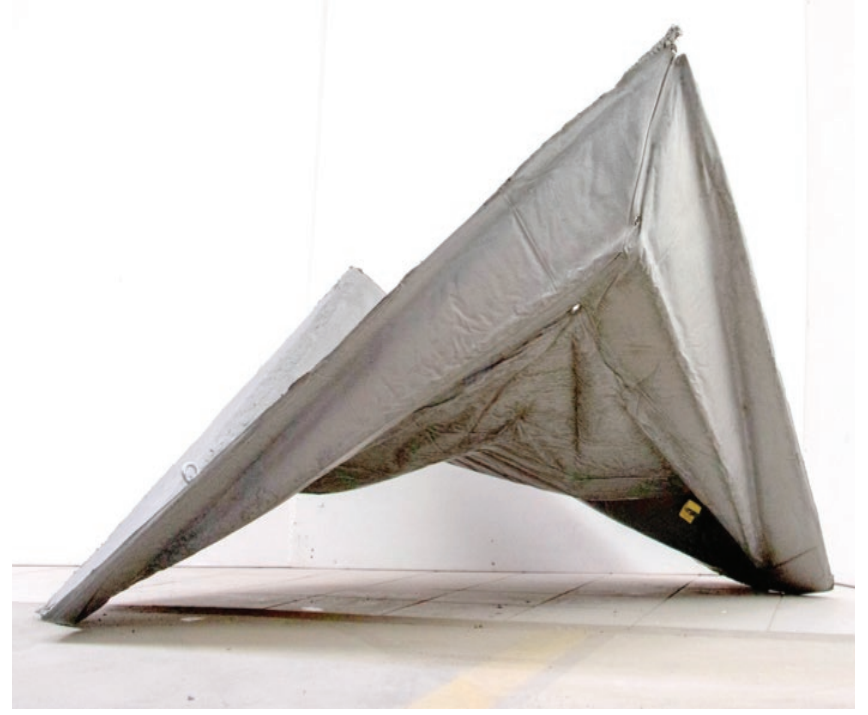

Figure $5.2 \mathrm{~m} \times 2 \mathrm{~m}$ proof of concept with $1 / 2{ }^{\prime \prime}$ thick creased shell form.

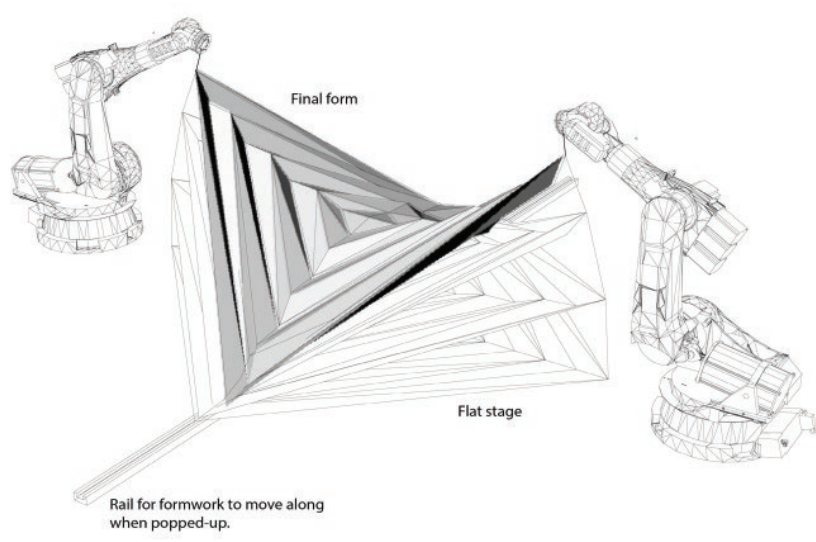

Figure 6. 'Creased shell' structure automated fabrication process with Robotic arms depicting flat formwork folding up into final geometry (drawing by APTUM). 